\title{
THE FACTORIZATION OF AN INTEGRAL MATRIX INTO A PRODUCT OF TWO INTEGRAL SYMMETRIC MATRICES ${ }^{1}$
}

\author{
BY OLGA TAUSSKY
}

Communicated on January 31, 1973

A theorem going back to Frobenius, but refound repeatedly (for references see [5], generalized to arbitrary fields states:

Every $n \times n$ matrix $A=\left(a_{i k}\right)$ with elements in a field $F$ is similar to its transpose $A^{\prime}$ :

$$
A^{\prime}=S^{-1} A S
$$

where $S$ can be chosen symmetric and with elements in $F$. This is equivalent to the fact that every $A$ can be expressed in the form

$$
A=S_{1} S_{2}
$$

when $S_{1}, S_{2}$ are symmetric, with elements in $F$ where $S_{1}$ is nonsingular.

Here a new concept is introduced. It is a form of degree $n$ associated with the matrix. ${ }^{2}$ For relation (1) implies

$$
S A^{\prime}=A S \text {. }
$$

This leads to a set of linear equations for the elements of the symmetric matrix $S$. If $A$ has all its roots different (though the general case leads to relevant results too) then the number of $F$-independent symmetric solutions $S$ of (3) is $n$. The elements of $S$ are then linear forms in $n$ parameters and det $S$ is a form of degree $n$ in $n$ variables. It is this form which plays an important role. If $A$ is replaced by a matrix similar to $A$ then $S$ undergoes a congruence transformation and the form is multiplied by a square factor in $F$.

For $n=2$ relation (3) leads to a single equation in 3 variables. This equation is given by

$$
a_{21} x_{1}+\left(a_{22}-a_{11}\right) x_{2}-a_{12} x_{3}=0
$$

AMS (MOS) subject classifications (1970). Primary 15A36, 12A50, $10 \mathrm{C} 10$.

Key words and phrases. Integral matrices.

${ }^{1}$ This work was carried out (in part) under an NSF contract; I have profited from discussions with E. Bender, J. Brillhart, E. C. Dade, D. Estes, H. Kisilevsky.

${ }^{2} \mathrm{~W}$. Givens informs me that he also considered such a form. 
if $S=\left(\begin{array}{cc}x_{1} & x_{2} \\ x_{2} & x_{3}\end{array}\right) \cdot{ }^{3}$ Apart from a multiple this form is given by

$$
\begin{aligned}
& a(\lambda, \mu)= \\
& \left(\alpha_{1} \alpha_{3}-\alpha_{2}^{2}\right) \lambda^{2}+\left(\alpha_{1} \beta_{3}-2 \alpha_{2} \beta_{2}+\alpha_{3} \beta_{1}\right) \lambda \mu+\left(\beta_{1} \beta_{3}-\beta_{2}^{2}\right) \mu^{2}
\end{aligned}
$$

where $\alpha_{1}, \alpha_{2}, \alpha_{3}$ and $\beta_{1}, \beta_{2}, \beta_{3}$ are a pair of independent solutions of (4). It can be shown that the discriminant of (5) and the discriminant of the form

$$
a_{21} x^{2}+\left(a_{22}-a_{11}\right) x y+a_{12} y^{2}
$$

differ by a square factor. The latter discriminant is also the discriminant of the characteristic polynomial of $A$.

The emphasis of the present research is on the case where $A$ in (2) is a rational integral matrix and on the question under what circumstances

$$
A=S_{1} S_{2}, \quad S_{i}=S_{i}^{\prime}, \quad S_{i} \text { with elements in } Z .
$$

For $n=2$ a number of results have been obtained in [6], [7], e.g.:

1. If $\operatorname{gcd}\left(a_{21},\left(a_{22}-a_{11}\right), a_{12}\right)=g$ then the discriminant of (5) and of (6) coincide apart from the factor $g^{2}$. Here the solutions $\alpha_{1}, \alpha_{2}, \alpha_{3}$; $\beta_{1}, \beta_{2}, \beta_{3}$ are assumed to be integral basis vectors for the lattice of all integral solutions of (4).

2. Let the characteristic polynomial of $A$ be $x^{2}-m$, when $m \equiv 2,3(4)$ and square free. Then (7) can only hold if the ideal class associated with $A$ by the relation

$$
A\left(\begin{array}{l}
\alpha_{1} \\
\alpha_{2}
\end{array}\right)=\alpha\left(\begin{array}{l}
\alpha_{1} \\
\alpha_{2}
\end{array}\right)
$$

is of order 1 or 2 or 4 . Here $\alpha$ is a characteristic root of $A$ and $\alpha_{1}, \alpha_{2} \in Z[\alpha]$ form a basis for an ideal $\mathfrak{U}$ in $Z[\alpha]$.

3. Relation (7) holds for $A$ if and only if the form (5) represents a factor $d$ of $m$ (the discriminant of (5) is $4 m$ ). In this case $-m / d$ is also represented so that in (7) one of the $S_{i}$ has determinant $d$, the other $-m / d$.

4. For an arbitrary $2 \times 2$ integral $A$ the following fact holds: relation (7) can be satisfied if and only if the form (5) can be transformed by a unimodular similarity to a form whose middle coefficient is trace $A$.

5. Every integral $2 \times 2$ matrix can be factored as in (7) when a suitable integral scalar matrix is added to it.

In the case of general $n$ and the characteristic polynomial $f(x)$ of $A$ irreducible the problem can be studied via a result obtained previously (see [4]) by several authors.

\footnotetext{
${ }^{3}$ Since (4) defines a plane in 3-space the null-space of the binary quadratic form attached to $A$ can be regarded as the intersection of the plane with the cone $x_{1} x_{3}-x_{2}^{2}=0$.
} 
If $A$ and $S$ in (1) are integral then $S=\left(\right.$ trace $\left.\lambda \alpha_{i} \alpha_{k}\right)$ where $\lambda \in Q(\alpha), \alpha$ a zero of $f(x)$ and $\alpha_{1}, \ldots, \alpha_{n}$ form a basis for the ideal $\mathfrak{U}$ constructed as in (8). From this follows that for $S$ to be integral it is necessary and sufficient that

$$
\lambda \in\left(\mathfrak{U}^{2}\right)^{\prime}=\left(\mathfrak{U} \mathfrak{U}^{\prime}: \mathfrak{U}\right),
$$

when ' denotes the complementary ideal.

For $S_{1}$ and $S_{2}$ to be integral in (7) it is necessary and sufficient that also

$$
\alpha \lambda^{-1} \in\left(\mathfrak{U}: \mathfrak{U}^{\prime}\right)
$$

The form in $n$ variables of degree $n$ mentioned at the start is connected with the norm form of an ideal (for this see Theorems 3, 4 in [3]). Some information concerning the order to which this ideal belongs can be seen from the greatest divisors of certain sets of elements in the matrix $A$.

One of the factors $S_{i}$ in (7) can be chosen unimodular if and only if the ideal class corresponding to $A$ in $Z[\alpha]$ coincides with the ideal class corresponding to $A^{\prime}$. If $Z[\alpha]$ is the maximal order this is only possible if this ideal class is of order 1 or 2 , (see [1], [2]).

\section{REFERENCES}

1. D. K. Faddeev, On the characteristic equations of rational symmetric matrices, Dokl. Akad. Nauk SSSR 58 (1947), 753-754. (Russian) MR 9, 270.

2. O. Taussky, On matrix classes corresponding to an ideal and its inverse, Illinois J. Math. 1 (1957), 108-113. MR 20 \#845.

3. - Ideal matrices. I, Arch. Math. 13 (1962), 275-282. MR 27 \# 168.

4. - On the similarity transformation between an integral matrix with irreducible characteristic polynomial and its transpose, Math. Ann. 166 (1966), 60-63. MR 33 \# 7355.

5. - Symmetric matrices and their role in the study of general matrices, Linear Algebra and Appl. 5 (1972), 147-159.

6. - The factorization of an integral matrix into a product of two integral symmetric matrices. I, Acta Arith. (to appear).

7. - The factorization of an integral matrix into a product of two integral symmetric matrices. II, Comm. Pure Appl. Math. (to appear).

Department of Mathematics, California institute of Technology, Pasadena, CALIFornia 91109 